\title{
Diabetes and Employment
}

\author{
American Diabetes Association
}

A s of 2010, nearly 26 million Americans have diabetes (1), most of whom are or wish to be participating members of the workforce. Diabetes usually has no impact on an individual's ability to do a particular job, and indeed an employer may not even know that a given employee has diabetes. In 1984, the American Diabetes Association adopted the following position on employment:

Any person with diabetes, whether insulin [treated] or non-insulin [treated], should be eligible for any employment for which he/she is otherwise qualified.

Questions are sometimes raised by employers about the safety and effectiveness of individuals with diabetes in a given job. When such questions are legitimately raised, a person with diabetes should be individually assessed to determine whether or not that person can safely and effectively perform the particular duties of the job in question. This document provides a general set of guidelines for evaluating individuals with diabetes for employment, including how an assessment should be performed and what changes (accommodations) in the workplace may be needed for an individual with diabetes.

\section{EVALUATING INDIVIDUALS WITH DIABETES FOR}

EMPLOYMENT-It was once common practice to restrict individuals with diabetes from certain jobs or classes of employment solely because of the diagnosis of diabetes or the use of insulin, without regard to an individual's abilities or circumstances. Such "blanket bans" are medically inappropriate and ignore the many advancements in diabetes management that range from the types of medications used to the tools used to administer them and to monitor blood glucose levels.

Employment decisions should not be based on generalizations or stereotypes regarding the effects of diabetes. The impact of diabetes and its management varies widely among individuals. Therefore, a proper assessment of individual candidates for employment or current employees must take this variability into account.

In addition, federal and state laws require employers to make decisions that are based on assessment of the circumstances and capabilities of the individual with diabetes for the particular job in question $(2,3)$. Application of blanket policies to individuals with diabetes results in people with diabetes being denied employment for which they are well qualified and fully capable of performing effectively and safely. It should be noted that, as a result of amendments to the Americans with Disabilities Act, which became effective on 1 January 2009, all persons with diabetes are considered to have a "disability" within the meaning of that law. This is because, among other reasons, diabetes constitutes a substantial limitation on endocrine system functioning-the Act was amended to extend its coverage to persons with a substantial limitation in, among other things, a major bodily function, such as the endocrine system. Therefore, persons with diabetes are protected from discrimination in employment and other areas. The amendments overturned a series of Supreme Court decisions that had severely narrowed who was covered by the law and resulted in many people with diabetes and other chronic illnesses being denied protection from discrimination. This section provides an overview of the factors relevant to a medically appropriate

Revised Fall 2009

DOI: $10.2337 / \mathrm{dcl} 2$-s094

(C) 2012 by the American Diabetes Association. Readers may use this article as long as the work is properly cited, the use is educational and not for profit, and the work is not altered. See http://creativecommons.org/ licenses/by-nc-nd/3.0/ for details.

individualized assessment of the candidate or employee with diabetes.

\section{Role of diabetes health care professionals}

When questions arise about the medical fitness of a person with diabetes for a particular job, a health care professional with expertise in treating diabetes should perform an individualized assessment. The involvement of the diabetes health care professional should occur before any adverse employment decision, such as failure to hire or promote or termination. A health professional who is familiar with the person with diabetes and who has expertise in treating diabetes is best able to perform such an assessment. In some situations and in complex cases, an endocrinologist or a physician who specializes in treating diabetes or its complications is the best qualified health professional to assume this responsibility (4). The individual's treating physician is generally the health care professional with the best knowledge of an individual's diabetes. Thus, even when the employer utilizes its own physician to perform the evaluation, the opinions of the treating physician and other health care professionals with clinical expertise in diabetes should be sought out and carefully considered. In situations where there is disagreement between the opinion of the employee's treating physician and that of the employer's physician, the evaluation should be handed over to an independent health care professional with significant clinical expertise in diabetes.

\section{Individual assessment}

A medical evaluation of an individual with diabetes may occur only in limited circumstances (3). Employers may not inquire about an individual's health statusdirectly or indirectly and regardless of the type of job-before making a job offer, but may require a medical examination or make a medical inquiry once an offer of employment has been extended and before the individual begins the job. 
The job offer may be conditioned on the results of the medical inquiry or examination. An employer may withdraw an offer from an applicant with diabetes only if it becomes clear that he or she cannot do the essential functions of the job or would pose a direct threat (i.e., a significant risk of substantial harm) to health or safety and such threat could not be eliminated with an accommodation (a workplace change that enables a worker with a disability to safely and effectively perform job duties). Another situation in which a medical evaluation is permissible is when a problem potentially related to the employee's diabetes arises on the job and such problem could affect job performance and/or safety. In this situation, a physician may be asked to evaluate the employee's fitness to remain on the job and/or his or her ability to safely perform the job.

Employers also may obtain medical information about an employee when the employee has requested an accomodation and his or her disability or need for accommodation is not obvious. An employer should not rely on a medical evaluation to deny an employment opportunity to an individual with diabetes unless it is conducted by a health care professional with expertise in diabetes and based on sufficient and appropriate medical data. The information sought and assessed must be properly limited to data relevant to the individual's diabetes and job performance (3). The data needed will vary depending on the type of job and the reason for the evaluation, but an evaluation should never be made based only on one piece of data, such as a single blood glucose result or A1C result. Since diabetes is a chronic disease in which health status and management requirements naturally change over time, it is inappropriateand medically unnecessary-for examiners to collect all past laboratory values or information regarding office visits whether or not related to diabetes. Only medical information relevant to evaluating an individual's current capacity for safe performance of the particular job at issue should be collected. For example, in some circumstances a review of an individual's hypoglycemia history may be relevant to the evaluation and should be collected.

Information about the individual's diabetes management (such as the current treatment regimen, medications, and blood glucose logs), job duties, and work environment are all relevant factors to be considered. Only health care professionals tasked with such evaluations should have access to employee medical information, and this information must be kept separate from personnel records (3).

\section{Screening guidelines}

A number of screening guidelines for evaluating individuals with diabetes in various types of high risk jobs have been developed in recent years. Examples include the American College of Occupational and Environmental Medicine's National Consensus Guideline for the Medical Evaluation of Law Enforcement Officers, the National Fire Protection Association's Standard on Comprehensive Occupational Medical Program for Fire Departments, the U.S. Department of Transportation's Federal Motor Carrier Safety Administration's Diabetes Exemption Program, and the U.S. Marshall Service and Federal Occupational Health Law Enforcement Program Diabetes Protocol.

Such guidelines and protocols can be useful tools in making decisions about individual candidates or employees if they are used in an objective way and based on the latest scientific knowledge about diabetes and its management. These protocols should be regularly reevaluated and updated to reflect changes in diabetes knowledge and evidence and should be developed and reviewed by health care professionals with significant experience in diabetes and its treatment. Individuals who do not meet the standards set forth in such protocols should be given the opportunity to demonstrate exceptional circumstances that would justify deviating from the guidelines. Such guidelines or protocols are not absolute criteria but rather the framework for a thorough individualized assessment.

\section{Recommendations}

- People with diabetes should be individually considered for employment based on the requirements of the specific job and the individual's medical condition, treatment regimen, and medical history. (E)

- When questions arise about the medical fitness of a person with diabetes for a particular job, a health care professional with expertise in treating diabetes should perform an individualized assessment; input from the treating physician should always be included. (E)

- Employment evaluations should be based on sufficient and appropriate medical data and should never be made based solely on one piece of data. (E)

- Screening guidelines and protocols can be useful tools in making decisions about employment if they are used in an objective way and based on the latest scientific knowledge about diabetes and its management. (E)

\section{EVALUATING THE SAFETY RISK OF EMPLOYEES WITH}

DIABETES - Employers who deny job opportunities because they perceive all people with diabetes to be a safety risk do so based on misconceptions, misinformation, or a lack of current information about diabetes. The following guidelines provide information for evaluating an individual with diabetes who works or seeks to work in what may be considered a safetysensitive position.

\section{Safety concerns}

The first step in evaluating safety concerns is to determine whether the concerns are reasonable in light of the job duties the individual must perform. For most types of employment (such as jobs in an office, retail, or food service environment) there is no reason to believe that the individual's diabetes will put employees or the public at risk. In other types of employment (such as jobs where the individual must carry a firearm or operate dangerous machinery) the safety concern is whether the employee will become suddenly disoriented or incapacitated. Such episodes, which are usually due to severely low blood glucose (hypoglycemia), occur only in people receiving certain treatments such as insulin or secretagogues such as sulfonylureas and even then occur infrequently. Workplace accommodations can be made that are minimal yet effective in helping the individual to manage his or her diabetes on the job and avoid severe hypoglycemia.

\section{Hypoglycemia}

Hypoglycemia is defined as a blood glucose level $<70 \mathrm{mg} / \mathrm{dl}(4,6)$. It is a potential side effect of some diabetes treatments, including insulin and sulfonlyureas. It can usually be effectively self-treated by ingestion of glucose (carbohydrate) and is not often associated with loss of consciousness or a seizure. Severe hypoglycemia, requiring the assistance of another person, is a medical emergency. 
Symptoms of severe hypoglycemia may include confusion or, rarely, seizure or loss of consciousness (6). Most individuals with diabetes never experience an episode of severe hypoglycemia because either they are not on medication that causes it or they recognize the early warning signs and can quickly self-treat the problem by drinking or eating. Also, with self-monitoring of blood glucose levels, most people with diabetes can manage their condition in such a manner that there is minimal risk of incapacitation from hypoglycemia because mildly low glucose levels can be easily detected and treated $(4,7)$.

A single episode of severe hypoglycemia should not per se disqualify an individual from employment. Rather, an appropriate evaluation should be undertaken by a health care professional with expertise in diabetes to determine the cause of the low blood glucose, the circumstances of the episode, whether it was an isolated incident, whether adjustment to the insulin regimen may mitigate this risk, and the likelihood of such an episode happening again. Some episodes of severe hypoglycemia can be explained and corrected with the assistance of a diabetes health care professional.

However, recurrent episodes of severe hypoglycemia may indicate that an individual may in fact not be able to safely perform a job, particularly jobs or tasks involving significant risk of harm to employees or the public, especially when these episodes cannot be explained. The person's medical history and details of any history of severe hypoglycemia should be examined closely to determine whether it is likely that such episodes will recur on the job. In all cases, job duties should be carefully examined to determine whether there are ways to minimize the risk of severe hypoglycemia (such as adjustment of the insulin regimen or providing additional breaks to check blood glucose levels).

\section{Hyperglycemia}

In contrast to hypoglycemia, high blood glucose levels (hyperglycemia) can cause long-term complications over years or decades but does not normally lead to any adverse effect on job performance. The symptoms of hyperglycemia generally develop over hours or days and do not occur suddenly. Therefore, hyperglycemia does not pose an immediate risk of sudden incapacitation. While over years or decades, high blood glucose may cause long-term complications to the nerves (neuropathy), eyes (retinopathy), kidneys (nephropathy), or heart, not all individuals with diabetes develop these long-term complications. Such complications become relevant in employment decisions only when they are established and interfere with the performance of the actual job being considered. Evaluations should not be based on speculation as to what might occur in the future. Job evaluations should take high blood glucose levels into account only if they have already caused long-term complications such as visual impairment that interfere with performance of the specific job.

\section{Aspects of a safety assessment}

When an individual with diabetes is assessed for safety risk there are several aspects that must be considered.

Blood glucose test results. A single blood glucose test result only gives information about an individual's blood glucose level at one particular point in time. Because blood glucose levels fluctuate throughout the day (this is also true for people without diabetes), one test result is of no use in assessing the overall health of a person with diabetes. The results of a series of self-monitored blood glucose measurements over a period of time, however, can give valuable information about an individual's diabetes health. Blood glucose records should be assessed by a health care professional with expertise in diabetes (7). History of severe hypoglycemia. Often, a key factor in assessing employment safety and risk is documentation of incidents of severe hypoglycemia. An individual who has managed his or her diabetes over an extended period of time without experiencing severe hypoglycemia is unlikely to experience this condition in the future. Conversely, multiple incidents of severe hypoglycemia may in some situations be disqualifying for high-risk occupations. However, the circumstances of each incident should be examined, as some incidents can be explained due to changes in insulin dosage, illness, or other factors and thus will be unlikely to recur or have already been addressed by the individual through changes to his or her diabetes treatment regimen or education.

Hypoglycemia unawareness. Some individuals over time lose the ability to recognize the early warning signs of hypoglycemia. These individuals are at increased risk for a sudden episode of severe hypoglycemia. Some of these individuals may be able to lessen this risk with careful changes to their diabetes management regimen (for example, more frequent blood glucose testing or frequent meals). Presence of diabetes-related complications. Chronic complications that may result from long-term diabetes involve the blood vessels and nerves. These complications may involve nerve (neuropathy), eye (retinopathy), kidney (nephropathy), and heart disease. In turn, these problems can lead to amputation, blindness or other vision problems, including vision loss, kidney failure, stroke, or heart attack. As these complications could potentially affect job performance and safety, such complications should be evaluated by a specialist in the specific area related to the complication. If complications are not present, their possible future development should not be addressed, both because of laws prohibiting such consideration and because with medical monitoring and therapies, long-term complications can now often be avoided or delayed. Thus, many people with diabetes never develop any of these complications, and those that do generally develop them over a period of years.

\section{Inappropriate assessments}

The following tools and terms do not accurately reflect the current state of diabetes treatment and should be avoided in an assessment of whether an individual with diabetes is able to safely and effectively perform a particular job.

Urine glucose tests. Urine glucose results are no longer considered to be an appropriate and accurate methodology for assessing diabetes control (8). Before the mid-1970s, urine glucose tests were the best available method of monitoring blood glucose levels. However, the urine test is not a reliable or accurate indicator of blood glucose levels and is a poor measure of the individual's current health status. Blood glucose monitoring is a more accurate and timely means to measure glycemic control. Urine glucose tests should never be used to evaluate the employability of a person with diabetes.

AIC and estimated average glucose (eAG). Hemoglobin AlC (A1C) test results reflect average glycemia over several months and correlate with mean plasma glucose levels (4). An eAG is directly related to A1C and also provides an individual with an estimate of average blood glucose over a period of time, but it uses the same values and units that are observed when using a glucose meter or 
recording a fasting glucose value on a lab report (5). A1C/eAG values provide health care providers with important information about the effectiveness of an individual's treatment regimen (4) but are often misused in assessing whether an individual can safely perform a job. Because they identify only averages and not whether the person had severe extreme blood glucose readings, AlC/eAG results are of no value in predicting shortterm complications of diabetes and thus have no use in evaluating individuals in employment situations.

The American Diabetes Association recommends that in most patients AlC levels be kept below 7\% (4), or eAG below $154 \mathrm{mg} / \mathrm{dl}$. This recommendation sets a target in order to lessen the chances of long-term complications of high blood glucose levels but does not provide useful information on whether the individual is at significant risk for hypoglycemia or suboptimal job performance and is not a measure of "compliance" with therapy. An AlC or eAG cut off score is not medically justified in employment evaluations and should never be a determinative factor in employment.

"Uncontrolled" or "brittle" diabetes. Sometimes an individual's diabetes is described as "uncontrolled," "poorly controlled," or "brittle." These terms are not well defined and are not relevant to job evaluations. As such, giving an opinion on the level of "control" an individual has over diabetes is not the same as assessing whether that individual is qualified to perform a particular job and can do so safely. Such an individual assessment is the only relevant evaluation.

\section{Recommendations}

- Evaluating the safety risk of employees with diabetes includes determining whether the concerns are reasonable in light of the job duties the individual must perform. (E)

- Most people with diabetes can manage their condition in such a manner that there is no or minimal risk of incapacitation from hypoglycemia at work. A single episode of severe hypoglycemia should not per se disqualify an individual from employment, but an individual with recurrent episodes of severe hypoglycemia may be unable to safely perform certain jobs, especially when those episodes cannot be explained. (E)

- Hyperglycemia does not pose an immediate risk of sudden incapacitation on the job, and long-term complications are relevant in employment decisions only when they are established and interfere with the performance of the actual job being considered. (E)

- Proper safety assessments should include review of blood glucose test results, history of severe hypoglycemia, presence of hypoglycemia unawareness, and presence of diabetes-related complications and should not include urine glucose or AIC/eAG tests or be based on a general assessment of level of control. (E)

\section{ACCOMMODATING EMPLOYEES WITH}

DIABETES - Individuals with diabetes may need certain changes or accommodations on the job in order to perform their work responsibilities effectively and safely. Federal and state laws require the provision of "reasonable accommodations" to help an employee with diabetes to perform the essential functions of the job (3). Additional laws provide for leave for an employee to deal with his or her medical needs or those of a family member (9). Although there are some typical accommodations that many people with diabetes use, the need for accommodations must be assessed on an individualized basis (2).

\section{Accommodating daily diabetes management needs}

Many of the accommodations that employees with diabetes need on a day-today basis are those that allow them to manage their diabetes in the workplace as they would elsewhere. They are usually simple accommodations, can be provided without any cost to the employer, and should cause little or no disruption in the workplace. Most employers are required to provide accommodations unless those accommodations would create an undue burden (3). Some accommodations that may be needed include the following.

Testing blood glucose. Breaks may be needed to allow an individual to test blood glucose levels when needed. Such checks only take minutes to complete. Some individuals use continuous glucose monitors but will still need an opportunity to check blood glucose with a meter. Blood glucose can be checked wherever the employee is without putting other employees at risk, and employers should not limit where employees with diabetes are permitted to manage their diabetes. Some employees may prefer to have a private location for testing or other diabetes care tasks that should be provided whenever feasible.

Administering insulin. Employees may need short breaks during the workday to administer insulin when it is needed. Insulin can be safely administered wherever the employee happens to be. The employee may also need a place to store insulin and other supplies if work conditions (such as extreme temperatures) prevent the supplies from being carried on the person (10).

Food and drink. Employees may need access to food and/or beverages during the workday. This is particularly important in the event that the employee needs to quickly respond to low blood glucose levels or maintain hydration if glucose levels are high. Employees should be permitted to consume food or beverages as needed at their desk or work station (except in an extremely rare situation in which this would pose a hazard and create a safety issue, and if this is the case, an alternative site should be provided).

Leave. Employees may need leave or a flexible work schedule to accommodate medical appointments or other diabetes care needs. Occasionally, employees may need to miss work due to unanticipated events (severe hypoglycemic episode) or illness.

Work schedules. Certain types of work schedules, such as rotating or split shifts, can make it especially difficult for some individuals to manage diabetes effectively.

\section{Accommodating complications of diabetes}

In addition to accommodating the day-today management of diabetes in the workplace, for some individuals it is also necessary to seek modifications for longterm diabetes-related complications. Such people can remain productive employees if appropriate accommodations are implemented.

For example, an employee with diabetic retinopathy or other vision impairments may benefit from using a big screen computer or other visual aids, while an employee with nerve pain may benefit from reduced walking distances or having the ability to sit down on the job. Individuals with kidney problems may need to have flexibility to take time off work for dialysis treatment.

It is impossible to provide an exhaustive list of potential accommodations. The key message in accommodating an employee with diabetes is to ensure that 
accommodations are tailored to the individual and effective in helping the individual perform his or her job. Input from health care professionals who specialize in the particular complication, or from vocational rehabilitation specialists or organizations, may help identify appropriate accommodations.

\section{Recommendations}

- Individuals with diabetes may need accommodations on the job in order to perform their work responsibilities effectively and safely; these include accommodating daily diabetes needs and, when present, the complications of diabetes. All such accommodations must be tailored to the individual and effective in helping the individual perform his or her job. (E)

CONCLUSION-Individuals with diabetes can and do serve as highly productive members of the workforce. While not every individual with diabetes will be qualified for, nor can perform, every available job, reasonable accommodations can readily be made that allow the vast majority of people with diabetes to effectively perform the vast majority of jobs. The therapies for, and effects of, diabetes vary greatly from person to person, so employers must consider each person's capacities and needs on an individual basis. People with diabetes should always be evaluated individually with the assistance of experienced diabetes health care professionals. The requirements of the specific job and the individual's ability to perform that job, with or without reasonable accommodations, always need to be considered.

Acknowledgments-The American Diabetes Assocation thanks the members of the volunteer writing group for this updated statement: John E. Anderson, MD; Michael A. Greene, JD; John W. Griffin, Jr., JD; Daniel B. Kohrman, JD; Daniel Lorber, MD, FACP, CDE; Christopher D. Saudek, MD; Desmond Schatz, MD; and Linda Siminerio, RN, PhD, CDE.

\section{References}

1. Centers for Disease Control and Prevention: National Diabetes Fact Sheet: General Information and National Estimates on Diabetes and Prediabetes in the U.S., 2011. Atlanta, GA, U.S. Department of Health and Human Services, Centers for Disease Control and Prevention, 2011
2. Equal Employment Opportunity Commission, "Questions and Answers About Diabetes in the Workplace and the Americans with Disabilities Act (ADA)" Oct. 29, 2003. Available from http://www .eeoc.gov/facts/diabetes.html. Accessed 26 May 2008

3. Americans with Disabilities Act of 1990, 42 U.S.C. $\S 12101$ et seq.

4. American Diabetes Association: Standards of medical care in diabetes-2012 (Position Statement). Diabetes Care 2012;35 (Suppl. 1): S76-S80

5. Nathan DM, Kuenen J, Borg R, Zheng H, Schoenfeld D, Heine R: Translating the AlC assay into estimated average glucose values. Diabetes Care 31: 1473-1478, 2008

6. American Diabetes Association: Defining and reporting hypoglycemia in diabetes, a report from the American Diabetes Association Workgroup on Hypoglycemia. Diabetes Care 28: 1245-1249, 2005

7. American Diabetes Association: Selfmonitoring of blood glucose (Consensus Statement). Diabetes Care 17: 81-86, 1994

8. American Diabetes Association: Tests of glycemia in diabetes (Position Statement). Diabetes Care 27 (Suppl. 1): S91-S93, 2004

9. Family Medical Leave Act of 1993, 29 U.S.C. $\S 2601$ et seq.

10. American Diabetes Association: Insulin administration (Position Statement). Diabetes Care 27 (Suppl. 1): S106-S109, 2004 